\title{
Génesis y período vital del Frontón Recoletos
}

\section{Recoletos Pelota Court, genesis and vital period}

\author{
C. López-González ${ }^{(*)}$, M. Carreiro-Otero ${ }^{(*)}$, J. García Navarro $^{(* *)}$
}

\section{RESUMEN}

El artículo analiza un singular paradigma arquitectónico, el Frontón Recoletos, fruto de la colaboración entre el arquitecto Secundino Zuazo y el ingeniero Eduardo Torroja. Se plantea describir el proceso de ideación, entre el proyecto inicial de abril de 1935 y la elaboración definitiva en septiembre del mismo año, así como ilustrar las permanencias y mutaciones de la obra durante la fase de ejecución. Del mismo modo, el relato de su ciclo vital ilustra la diversidad de usos que acogió. Dañado durante la guerra civil, se reconstruye para desaparecer en 1973. Se pierde así una de las piezas más significativas de la arquitectura española contemporánea. El texto muestra la colaboración entre dos disciplinas que, mediante sucesivas aproximaciones, transforman substancialmente el espacio arquitectónico fusionando armónicamente forma, función y estructura, a la par que pone de relieve la naturaleza versátil de una pieza que fue ideada con un marcado carácter de especialización.

Palabras clave: Frontón Recoletos; Torroja; Zuazo; estructura; espacio arquitectónico.

\section{ABSTRACT}

The Recoletos Pelota Court is a unique model of architectural harmony, created in collaboration by the architect Secundino Zuazo and the engineer Eduardo Torroja. This paper examines the evolution of the Recoletos project from its initial conception in April 1935 to the finalisation of the plans in September of the same year, how the design was implemented and adapted during the phase of construction, and the variety of uses acquired by the building over the course of its lifetime. Though restored after the damage suffered during the Spanish civil war, the structure was finally demolished in 1973, resulting in the loss of one of the most important buildings in contemporary Spanish architecture. The study explores the collaboration between two different disciplines and their successive refinements of the design to achieve an intimate harmony of form, function and structure that transformed the architectural space of the building. Finally, it illustrates the ultimate versatility of a structure conceived for a specific use.

Keywords: Recoletos Pelota Court; Torroja; Zuazo; structure; architectural space.

(*) ETS de Arquitectura - Universidade da Coruña. (España).

(**) giSCI - Universidad Politécnica de Madrid, (España).

Persona de contacto/Corresponding author: candido.lopez@udc.es (C. López-González)

Cómo citar este artículo/Citation: López-González, C., Carreiro-Otero, M., García-Navarro, J. (2014). Génesis y período vital del Frontón Recoletos. Informes de la Construcción, 66(536): e040, doi: http://dx.doi.org/10.3989/ic.14.060.

Licencia/License: Salvo indicación contraria, todos los contenidos de la edición electrónica de Informes de la Construcción se distribuyen bajo una licencia de uso y distribución Creative Commons Reconocimiento no Comercial 3.o. España (cc-by-nc). 


\section{PRECEDENTES EN LA TRADICIÓN DE UNA OBRA CONTEMPORÁNEA}

Si un espacio deportivo a cubierto representa el medioevo, este es la sala a cubierto para el juego de la pelota, el denominado Jeu de Paume. Su nombre se lo debe a les paumiers, artesanos parisinos especializados en la fabricación de bolas con un bote excepcional. La palma, que había sido practicada al aire libre se traslada en el siglo XIV a un espacio a cubierto especializado (1).

La consolidación del mismo se produce en el siglo de la Ilustración. Denis Diderot, editor de la enciclopedia francesa (2) recoge en el Septieme livraison, o Huitieme volume, de 1771, nueve láminas que describen el juego de la pelota, desde los elementos que permiten su práctica hasta los espacios físicos en los que tiene lugar.

En el siglo XIX, que alumbra el nacimiento de la reglamentación, se formalizan los diferentes deportes. Durante su última década y las dos primeras del siglo XX se contempla la expansión en el mundo entero del deporte de la pelota, concretamente el de la pelota vasca. En paralelo, el empleo del hierro y el hormigón armado abre el abanico a nuevas consideraciones en la expresión formal de las piezas deportivas (3), y posibilita la realización de numerosos frontones en las urbes en proceso de expansión.

Madrid se presenta como paradigma en la implantación en su ámbito edificado de diversos frontones en el tránsito entre los siglos XIX y XX (4). Desde el Jai Alai, en 1891, hasta que en 1936 se inaugura el Frontón Recoletos se erigen un nutrido grupo de frontones: el Fiesta Alegre, el Madrileño, el Retiro y el Buenos Aires en 1892. En 1893 se levantan el Euskal Jai, el de San Francisco el Grande y el Beti Jai, último de los frontones de cancha descubierta.

La celebración, a mayores de la actividad deportiva, de un nutrido número de actos sociales y la necesidad de independizar su realización de las inclemencias del tiempo generan la aparición de una estructura para cubrir la cancha. En 1895 una cubierta metálica completará el frontón Jai Alai y en 1899 se construirá el Central.

Las continuas exigencias de confort provocan que en 1922 surja el nuevo frontón Jai Alai, y en 1928, el Madrid. El broche final de esta secuencia de salas destinadas al deporte de la pelota vasca se cierra con la construcción del Frontón Recoletos, fruto de la colaboración entre el arquitecto Secundino Zuazo Ugalde, y el ingeniero de caminos Eduardo Torroja Miret.

El texto se propone desentrañar la complejidad del Frontón mediante el estudio de los procesos de ideación y construcción así como de su ciclo vital.

\section{LA COLABORACIÓN ENTRE EL ARQUITECTO SECUNDINO ZUAZO Y EL INGENIERO EDUARDO TORROJA: EL EQUILIBRIO ARMÓNICO}

La arquitectura y la ingeniería deslindan sus competencias profesionales en España, con la fundación de la Escuela de Canales y Caminos en Madrid, en 1802. La creación de las Escuelas de Arquitectura, de Madrid en 1844 y de Barcelona en 1868, refuerza la disociación en las aptitudes que se han de asignar a ambas profesiones. Mientras en la segunda mi- tad del XIX, los arquitectos se mostraban más atentos a las corrientes estilísticas, los ingenieros exploraban las innovaciones tecnológicas.

En las dos primeras décadas del siglo XX se escuchan voces que propugnan el trabajo conjunto entre las dos figuras profesionales. El ingeniero Vicente Machimbarrena Gogorza, profesor de la Escuela de Caminos, Canales y Puertos, ilustra esta actitud. En 1924 manifiesta con relación al proyecto del concurso de 1901 para la construcción de un puente en la ciudad de Bilbao que "para llegar a este resultado (que estructura y forma se fundan) es indispensable que desde la primera concepción de la obra artística (un puente), actúen invariablemente unidos ingeniero y arquitecto» (5).

En este contexto, aparece en escena la figura del madrileño Torroja (1899-1961) (6). Entre sus profesores figuran los ingenieros V. Machimbarrena y José Eugenio Ribera Dutasta. Este último funda la Compañía de Construcciones Hidráulicas Civiles, en la cual Torroja entra a trabajar en 1923, al finalizar sus estudios de ingeniería.

Torroja crea en 1927 su propia Oficina de Proyectos, y a principios de los años 30 la empresa ICON -Investigaciones de la Construcción- y lidera un grupo de profesionales, arquitectos e ingenieros de caminos, que en 1934 funda el hoy denominado IETcc -Instituto de Ciencias de la Construcción Eduardo Torroja-.

Editor entre 1934 y 1936 de la revista Hormigón y Acero (7), despliega las facetas de proyectista e ingeniero constructor, colaborando con los más destacados arquitectos racionalistas madrileños (8): con el equipo coordinado por Modesto López Otero en la Ciudad Universitaria de Madrid, con Manuel Sánchez Arcas en el Mercado de Algeciras, con Carlos Arniches Moltó y Martín Domínguez Esteban en el Hipódromo de la Zarzuela, y finalmente con Zuazo en el Frontón Recoletos.

Como resultado de sus cálculos estructurales (9) para la cubierta del Frontón, la Real Academia de Ciencias Exactas, Físicas y Naturales publica en 1942 el libro Comprobación y comportamiento de una estructura laminar.

Zuazo (1887-1970), arquitecto de origen vasco, desarrolla múltiples facetas en su vida profesional: promueve sus propias obras, asesora grupos bancarios extranjeros, dirige empresas e incluso, preside diversas inmobiliarias.

Colabora siendo estudiante con el arquitecto bilbaíno Emiliano Amann Amann, descubriendo el tradicionalismo, la monumentalidad y el academicismo de los maestros locales vascos que le dejarán grabada una impronta que no olvidará.

Profesional independiente desde 1920, en la década y media siguiente afronta los encargos más relevantes de una longeva vida profesional. La obra del Café Zahara en Madrid en 1927 anuncia sus preocupaciones arquitectónicas. Entre ellas, el empleo de la bóveda en la resolución del espacio interior (10).

Las primeras colaboraciones entre Zuazo y Torroja se producen en 1932 con dos realizaciones: el proyecto y obra de los Nuevos Ministerios en La Castellana y el Viaducto sobre la calle de Segovia, ambos en Madrid (11). De su entendimiento va a surgir en 1935 la obra cumbre de su producción arquitectónica: el Frontón Recoletos (12). 


\section{LOS PROCESOS DE IDEACIÓN Y CONSTRUCCIÓN: GÉNESIS DEL FRONTÓN RECOLETOS}

Las palabras con las que Zuazo fija, en sus Memorias (13) el origen del encargo del proyecto del Frontón Recoletos remiten a las conversaciones que, a raíz de su amistad con los pelotaris de Madrid, mantiene con uno de ellos interesándose por el conflicto existente entre los deportistas y el empresario de otro frontón, el Jai Alai.

Durante el transcurso de las mismas, el polifacético arquitecto adquiere el compromiso de construir un nuevo frontón y para ello constituye una sociedad, NEFSA -Nueva Empresa de Frontones, Sociedad Anónima-, la cual preside. La primera tarea que esta sociedad mercantil emprende es la búsqueda de un solar en el cual ubicarlo.

Con la identificación en el Paseo de Recoletos de una parcela propiedad en los años treinta del duque de Manzanedo (14) se inicia un proceso que desemboca en la construcción de una de las obras más significativas de la arquitectura española contemporánea.

\subsection{El lugar}

La parcela, ubicada en el borde nordeste del Madrid de los Austrias, adquiere una destacada presencia cuando Carlos María de Castro proyecta en 1857 el ensanche urbano, y el eje Norte-Sur, Prado-Recoletos-Castellana, transformándose en un elemento de nueva centralidad entre el viejo Madrid y el barrio de Salamanca. Esta posición se refuerza por la inserción en 1866 del monumental edificio de Museos, Archivos y Biblioteca Nacional, obra de Francisco Jareño de Alarcón, finalizado en 1892.

El derribo del Palacio de don Ramón Calderón que ocupaba desde mediados del XIX dicha parcela y su consiguiente subdivisión mediante el trazado de una calle particular paralela al Paseo de Recoletos, establecen los límites definitivos del solar.

Solar en esquina, con sus frentes urbanos a las calles Villanueva y del Cid, y con los linderos interiores conformados por una medianería y la citada calle particular. De formato trapezoidal, alcanza los 3.525,95 metros cuadrados.

Topográficamente, la calle del Cid y la calle particular se mantienen prácticamente horizontales, mientras que la calle Villanueva presenta un desnivel entre los puntos extremos del solar de dos metros aproximadamente.

El equipamiento deportivo a cubierto que se pretende construir en el solar se enfrenta en su entorno con un notable número de problemas: la ausencia de un espacio público contiguo que resuelva de manera eficiente el desalojo de los espectadores, y que a la vez dignifique el edificio con un vacío urbano; el marcado carácter residencial de la manzana, y consecuentemente la aparición de medianeras; la presencia en su lado norte de la contundente fachada lateral del mencionado edificio de Museos, Archivo y Biblioteca Nacionales; y finalmente, la diferencia de cota existente en la calle Villanueva.

La pieza del deporte va a resolver, mediante su integración formal y funcional, los inconvenientes de articulación urbana detectados en este lugar.

\subsection{La ideación inicial: el proyecto de abril de 1935}

La elaboración del primer proyecto del Frontón Recoletos, fechado en abril de 1935, establece con precisión los elementos formales, funcionales y estructurales con los cuales Zuazo y Torroja organizan la pieza arquitectónica (15).

La solución formal de descomponer los diferentes volúmenes del proyecto evidencia la voluntad de expresar las diversas actividades que se desarrollan en el objeto arquitectónico. Al gran volumen, coronado con dos láminas de hormigón asimétricas, que resuelve el espacio destinado al deporte, se incorporan dos volúmenes prismáticos menores con cometidos complementarios.

La aparente simetría adoptada en la distribución de las masas volumétricas se altera con la inserción de un edificio de viviendas en la superficie del solar no ocupada por el mismo. La disposición fraccionada de los volúmenes propone la esquina como principal nexo funcional con la trama urbana. El punto de intersección entre las calles Villanueva y del Cid juega un doble papel: se afirma como el principal lugar de acceso además de establecer la cota de referencia de la cancha de juego.

La organización en planta se confía a un trazado ortogonal que adopta la direccionalidad de la calle Villanueva como eje compositivo principal, matizado por las ligeras inflexiones angulosas de las gradas. Así mismo, se detecta en la planta del nivel de paseo de cancha la presencia formal de elementos verticales soporte de la planta de palcos, con un aspecto circular (Figura 1). Inflexiones y soportes se transformarán durante el proceso de ideación.

En la fachada a la calle Villanueva, el retranqueo del cuerpo principal genera un vacío, un espacio libre arbolado destinado a jardín, cerrado al ambiente urbano mediante la construcción de un frente continuo de planta baja. La asimetría de la cancha, la orientación, la lateralidad -la pelota se arroja contra un frontis, el frontón, existiendo una pared de rebote lateral y otra posterior- conjuntamente con la disposición del graderío conforman el espacio.

La expresividad en la imagen del Frontón se configura en el diálogo permanente entre la nueva experimentación formal (16) de las láminas de hormigón armado de la cubierta y el lenguaje vernacular, en el mejor sentido de la tradición de las fachadas, hacia la escena urbana. Imagen equilibrada entre modernidad y clasicismo (17).

En la cubierta se encuentra uno de los elementos de mayor significación y relevancia de la obra arquitectónica (18). La construcción de su estructura mediante una doble lámina cilíndrica de hormigón armado de 8 centímetros de espesor y 55 metros de luz a lo largo de las generatrices y 32,50 metros de anchura entre los bordes, conformada por dos arcos de circunferencia de 12,20 y 6,40 metros de radio respectivamente, enlazados por una directriz en forma de gaviota, se conforma en sí misma como el elemento de cierre y el configurador del espacio, definiendo el volumen de la sala.

El empleo de las láminas evita la aparición de las tan recurridas cerchas metálicas, cuyo uso implicaría la disposición de una doble carcasa, la exterior con una función de protección y la interior con un acabado formal. 


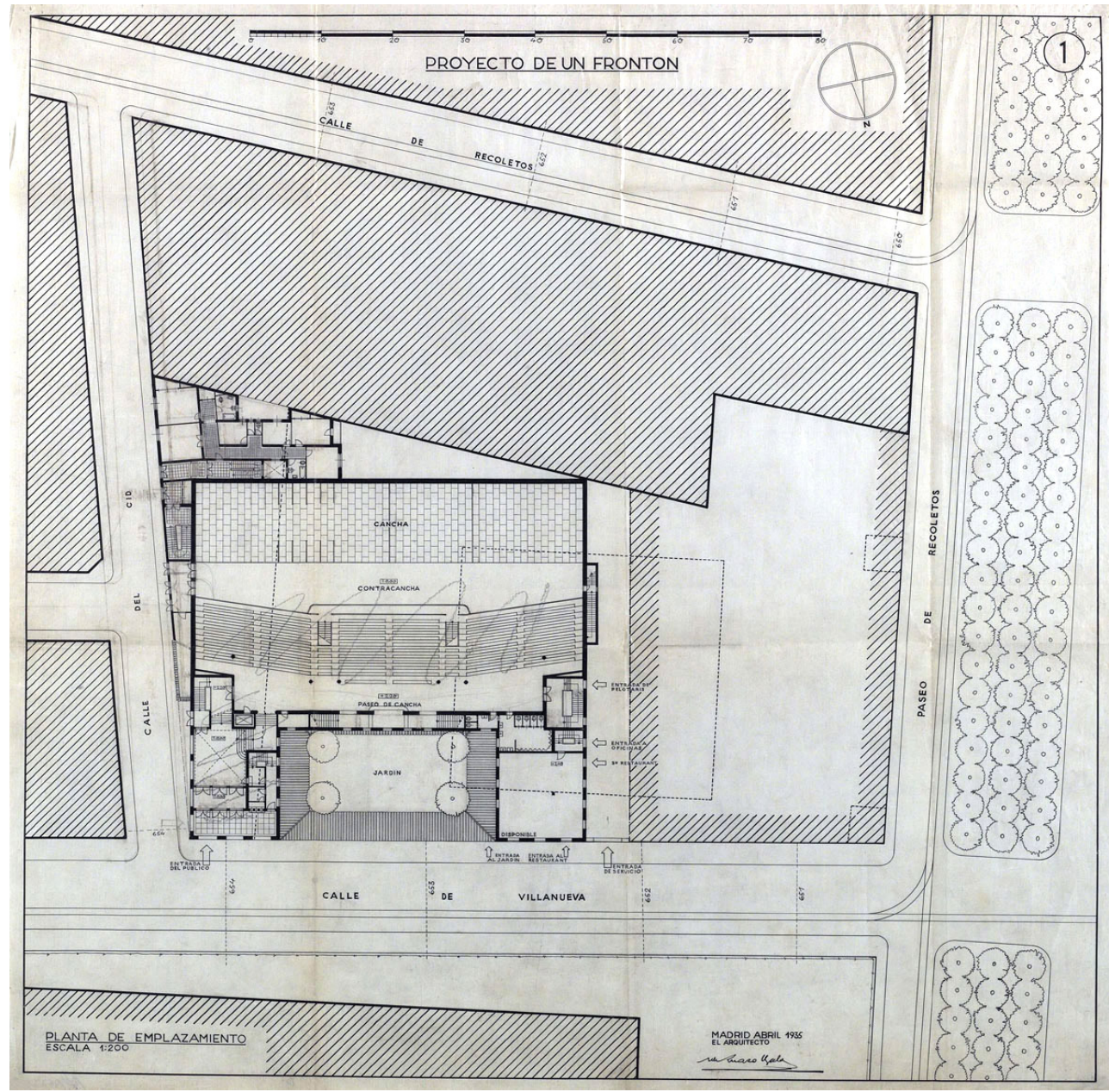

Figura 1. Proyecto de abril de 1935. Planta del nivel de paseo de cancha. (Fuente: Fondo Zuazo, Biblioteca Nacional de España)

Las bóvedas generadas, asimétricas, acogen dos lucernarios con orientación norte que proporcionan luz natural homogénea. La necesidad de iluminación y de una mínima altura de techo para la práctica deportiva se reflejan en la secuencia de las posibilidades estructurales exploradas. El vigor estructural resuelve definitivamente la forma de la cubierta, consiguiendo una diáfana expresividad estética (19).

Racionalismo y poética orgánica, funcionalidad y organicismo, libres de prejuicios formales y consideraciones estilísticas contaminadas. El arquitecto Juan Daniel Fullaondo Errazu explora una comprensión de mayor profundidad que aquella vinculada a una arquitectura racional, reivindicando para la pieza deportiva una posición de vanguardia (20).

Lo moldeable, lo adaptable, lo continuo y una oculta textura característica confieren el valor expresivo a la experimentación formal (21), relacionada con el empleo del hormigón armado. Una nueva estética aparece en la escena arquitectónica.

La asimetría de las láminas estructurales de la cubierta adquiere un mayor relieve con la disposición de dos lucernarios. Lucernarios con predominio de su traza longitudinal, sutilmente diferentes en su dimensión transversal, materializados mediante una celosía de elementos translúcidos, formados a partir de triángulos equiláteros de 140 centímetros de lado. La luz natural homogénea que inunda el espacio desde lo alto despeja de sombras e incómodos deslumbramientos el ámbito de la práctica deportiva, determinando la levedad del espacio (22). Del mismo modo, con relación a las condiciones de audición, la posición de los lucernarios garantiza un notable confort acústico.

La pureza formal en la cubierta induce a acomodar la luz artificial en el interior de los triángulos de la celosía, ocultando los mecanismos de iluminación. Celosía, con un doble acristalamiento exterior e interior. La apertura de este último permite el acceso a los reflectores para su control y mantenimiento.

\subsection{La propuesta definitiva: el proyecto de septiembre de 1935}

Permanencias y mutaciones, invariantes y transformaciones en la configuración espacial del proyecto, protagonizan el período que transcurre desde abril a septiembre del año 1935, formalizándose un segundo y definitivo proyecto.

Mientras las láminas de hormigón armado de la cubierta se ratifican como el elemento estructural significativo del proyecto, la sección se transforma mediante la incorporación de una estructura con una doble viga de gran canto que libera de soportes interiores la planta del nivel de paseo de cancha, reconfigurando el trazado de los diferentes niveles del graderío. 
La organización de la volumetría del proyecto precedente se mantiene: el volumen principal de la cancha de juego conserva su posición y su remate con el doble elemento laminar de hormigón armado, y los dos volúmenes menores permanecen en su ubicación previa. Sin embargo, un ligero pero contundente desplazamiento en la calle del Cid de uno de los dos volúmenes menores singulariza la esquina.

Este desequilibrio de las piezas menores refleja en el exterior la asimetría de la disposición interna de la pista de juego y el graderío, y señala el volumen del espacio propiamente deportivo. Volumen que con sus formas lobulares adquiere un protagonismo propio en su fachada a la calle del Cid, al desaparecer el edificio de viviendas proyectado inicialmente en la parte posterior del solar.

La nueva estructura interna genera el encadenamiento en los trazados de los graderíos mediante diversas curvaturas. La continuidad de las gráciles y livianas curvas, en relación con la doble forma laminar de la cubierta, dotan al espacio interno de una expresividad formal unitaria de la que hasta el momento carecía. La curvatura en forma de arco de la planta más noble, la planta de palcos, se refleja en la fachada a la calle del Cid a través de un mirador curvo (Figura 2).
El equilibrio entre la modernidad y el clasicismo de la imagen del Frontón Recoletos en su ideación inicial (23), se replantea con sutiles modificaciones: en la esquina, la reubicación de las taquillas de venta de entradas provoca la disposición de accesos funcionalmente especializados; en la fachada de la calle del Cid, los huecos de iluminación de la escalera se acomodan a su nuevo desarrollo, el balcón de la primera planta se unifica y las ventilaciones de la planta bajo cubierta adquieren una mayor dimensión disminuyendo en número; en la fachada a la calle Villanueva, se conserva la simetría de la composición pero se reduce el número de huecos, se redistribuyen y en los dos extremos se ajustan al nuevo trazado de las escaleras.

Asimismo, en el frente de la calle Villanueva el vacío central destinado a terraza, con un uso de restaurante de verano, difumina la percepción de un frente construido continuo como límite urbano. Esto posibilita la incorporación de una planta de semisótano con un uso público bajo la terraza y provoca la desaparición del arbolado del primer proyecto.

La disposición de la doble lámina cilíndrica con la transmisión de los principales esfuerzos estructurales a los muros laterales del Frontón, descargando los muros longitudina-

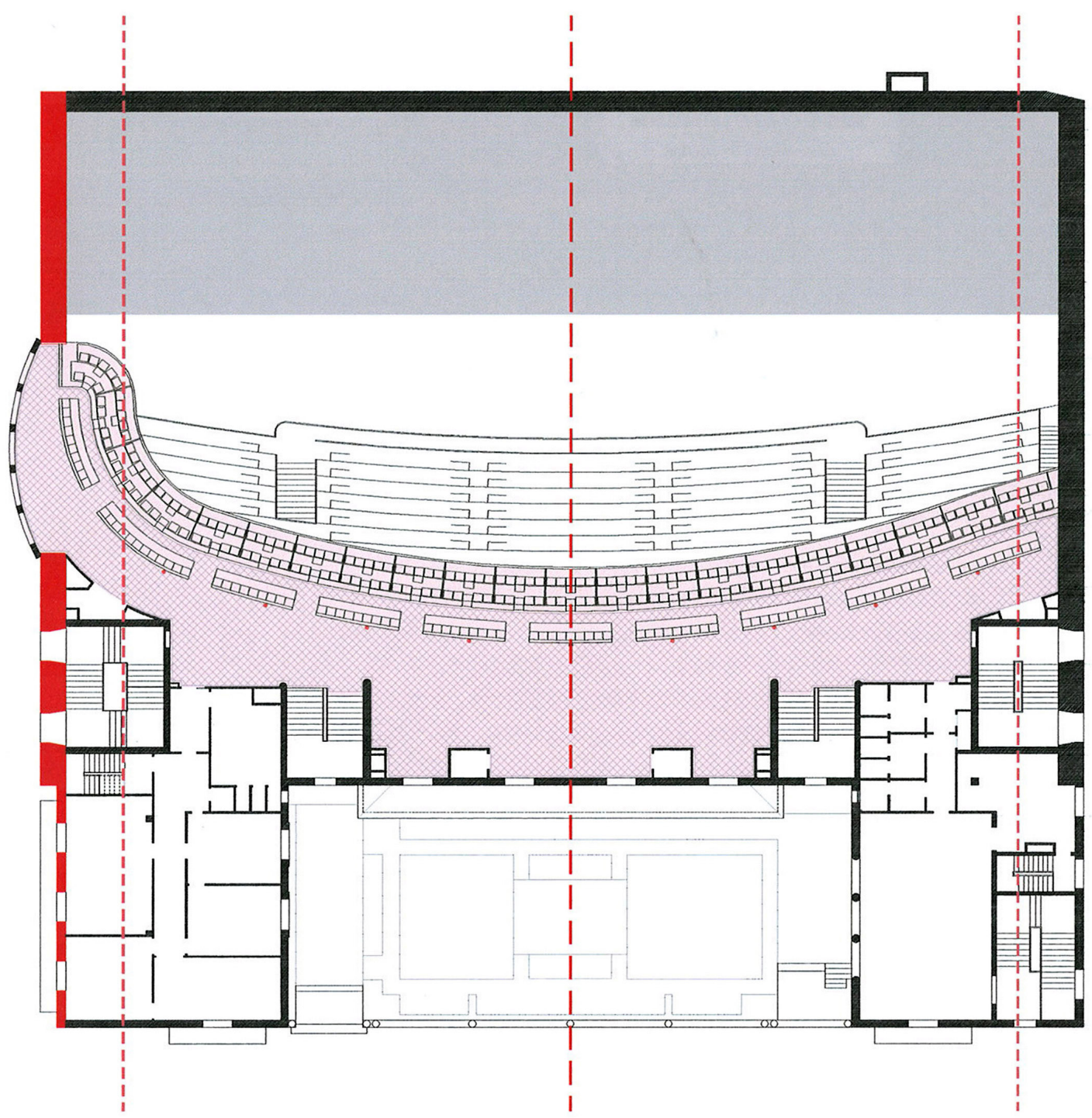

Figura 2. Análisis de la asimetría en la planta del nivel de palcos. Proyecto de septiembre de 1935 (dibujo C. López). 
les, permite una incorporación estructural (24) en la sección.

La ausencia de los soportes en la planta del paseo de cancha evidencia la introducción de esta nueva estructura que inserta dos vigas de gran canto paralelas a la fachada, separadas entre sí 4,50 metros, con unas ménsulas transversales de las cuales cuelga la planta de palcos mediante nueve tirantes péndolas, liberando el paseo de cancha de «incómodos» pilares. Las vigas de 3,50 metros de altura, arriostradas entre ellas, salvan una luz de 33,00 metros: 22,00 entre las pantallas de hormigón que conforman las escaleras de acceso a la planta de palcos, a los que se añaden dos vuelos laterales de 5,50 metros, uno a cada lado de las pantallas. Las cajas de las escaleras que dan acceso al nivel alto del graderío completan esta solución estructural con unas vigas en voladizo desde sus muros de hormigón (25).
La distribución de la estructura interna de las ménsulas incorpora su propio orden geométrico, con una rítmica distribución entre las dos cajas de escaleras, superponiéndose a la malla ortogonal primigenia. Las diversas geometrías presentes se adecuan en cada caso a las necesidades suscitadas con el funcionamiento del edificio, salvando la rigidez dimensional del módulo (Figura 3).

La exigencia de la máxima concentración posible en el punto focal de la actividad deportiva empuja a negar la luz natural lateral para no perturbar la acción del juego. Luz natural que resuelve la iluminación del resto de los espacios de modo convencional.

El objeto arquitectónico recupera en su interior el espacio público urbano, calles y plazas, reinterpretándolo. La significativa denominación de "paseo de cancha» con la cual se

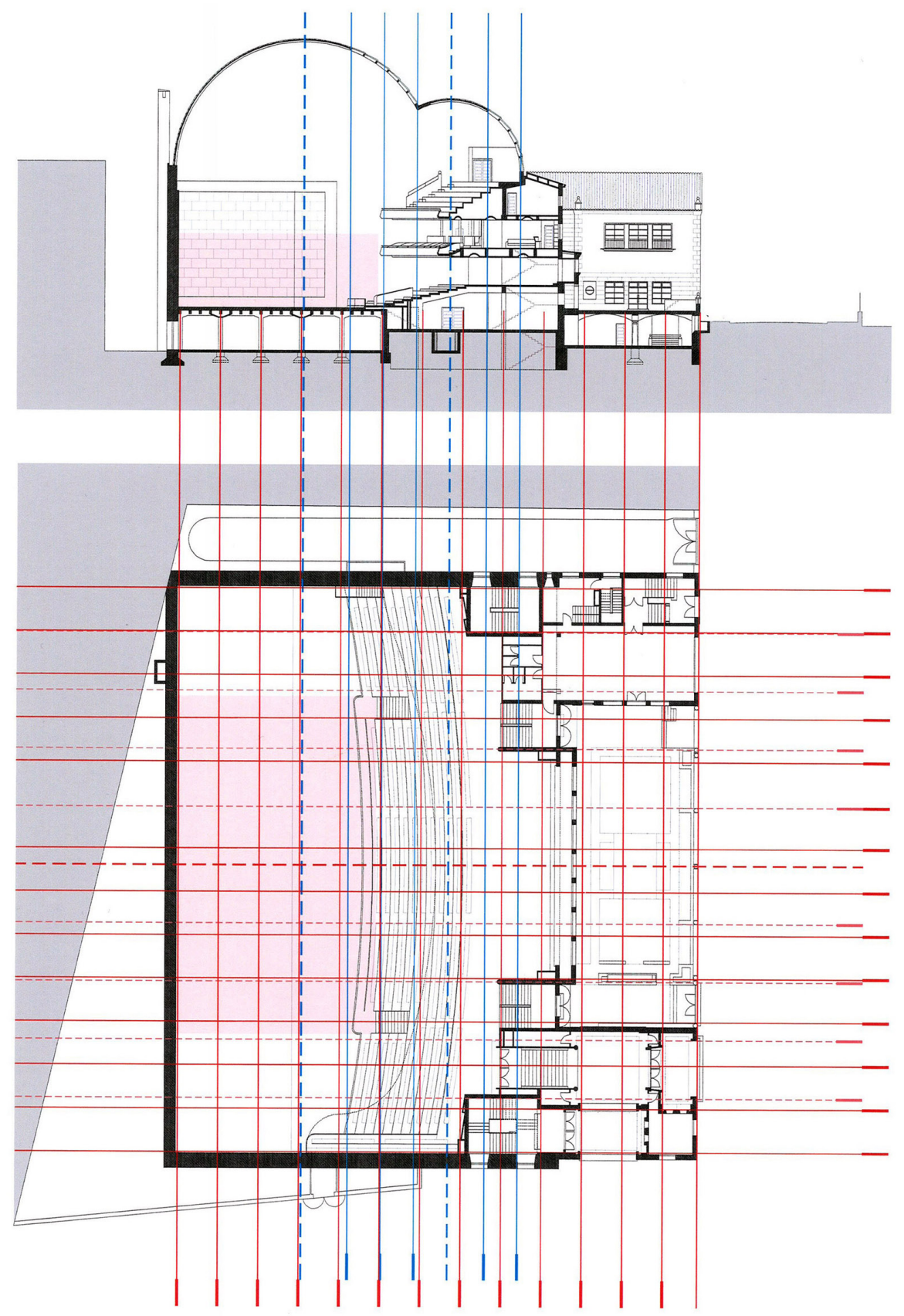

Figura 3. Órdenes estructurales y geometrías. Proyecto de septiembre de 1935 (dibujo C. López). 
designa el espacio posterior de las gradas inferiores constata esta relación.

Edificio singular, hito urbano, arquitectura a la que la colaboración entre Zuazo y Torroja dota de una esencialidad expresiva. Referencia urbana que encuentra en los planos ciegos de las fachadas el soporte para las expresiones artísticas de una plástica pictórica que el pintor vasco, Juan de Aranoa y Carredano incorpora a la particular pieza deportiva.

Claridad, lógica, rigor dimensional, son atributos que administrados con la incorporación de la intuición, lo sensitivo, la sugerencia, se postulan como los elementos clave de ideación en un elemento de múltiples escalas. Ideación a través de formas sencillas, depuradas, que buscan la esencia a través del manejo sensible de lo sustancial.

\subsection{Ejecución y fin de los trabajos}

Aunque el proyecto definitivo data de septiembre de 1935 , los trabajos de construcción del Frontón Recoletos se inician con anterioridad, en agosto del mencionado año. Una fotografía del 5 de septiembre, que refleja el estado de ejecución del encofrado del piso de cancha, indica que el movimiento de tierras y la cimentación ya se habían realizado previamente.

La construcción de la obra se confía a la empresa Huarte y Cía, S. L. Construcciones fundada y presidida por Félix Huarte Goñi, empresario industrial navarro y miembro del Consejo de Administración de NEFSA. La notable sintonía entre Zuazo y Mario Arlegui, «el buen encargado de la Casa Huarte, con el que me entendí para construir la difícil estructura de hormigón armado del Frontón Recoletos» (26), genera rapidez en la realización de los diferentes tajos durante los siguientes meses del invierno de 1935 (27), terminándose en febrero de 1936 .

Simultáneamente al corto espacio de tiempo del proceso de construcción se produce un hecho que, sin modificar aspectos fundamentales del proyecto, provoca cambios en la ejecución de la obra: NEFSA, la empresa propietaria, le solicita a dos especialistas en estructuras, los ingenieros J. E. Ribera y José María Aguirre Gonzalo, un informe acerca de la viabilidad de la estructura laminar de la cubierta (28).

El informe que emiten recoge diversas observaciones. La principal, que afecta a la formalización y percepción del espacio, deriva de necesidades estructurales, y aconseja elegir entre prolongar las claraboyas hasta los tímpanos haciéndolas continuas en la dirección de las generatrices de uno a otro lado de los muros laterales, o fraccionarlas con un menor tamaño, incorporando la membrana que forma la bóveda entre ellas, al objeto de evitar el peligro que pudiesen ocasionar esfuerzos secundarios.

Torroja, que en el proyecto había interrumpido ambos lucernarios al disponer una faja maciza de lámina en contacto con los muros laterales, escoge prolongarlos y construirlos en toda la longitud de la luz estructural. La incorporación de más luz cenital enfatiza la dimensión longitudinal de la sala, realzando su naturaleza liviana.

El resto de las observaciones las consideran pertinentes y se incorporan: la construcción de una cimbra horizontal con las contraflechas correspondientes; la realización de la maniobra del descimbrado de manera suave y el control de las deformaciones producidas al efectuar dicha operación; y la incorporación de un aislante térmico exterior para evitar las oscilaciones térmicas del hormigón armado de las láminas.

Durante el mes de noviembre de 1935, al objeto de cumplir con la indicación de controlar las deformaciones, se le solicita a ICON una propuesta completa de instalación de control. Una vez se formula, esta se lleva a cabo de modo inmediato, en el siguiente mes, con la realización del descimbrado. La cubierta se descimbra en un tiempo récord, tres meses y medio desde los trabajos de cimentación.

En paralelo, se realiza a propuesta de NEFSA un modelo reducido a escala $1 / 10$ lo cual permite ensayar, desde el 10 de noviembre al 5 de diciembre, con cargas similares a las condiciones reales y adoptar las correspondientes medidas correctoras.

En relación a la última de las observaciones mencionadas, los proyectistas, que ya habían considerado la instalación de un revestimiento interior de las láminas de hormigón armado con planchas de corcho para establecer una acústica adecuada en el espacio del deporte, incorporan una capa de corcho por encima de las mismas.

La obra, ejecutada con una inusitada velocidad, no se concluye en su totalidad. La realización del volumen prismático que se dispone en la calle Villanueva con la esquina a la calle particular se pospone.

Y aun cuando el Frontón Recoletos presenta partes inacabadas (Figura 4), su inauguración se produce el 29 de febrero de 1936, año bisiesto.

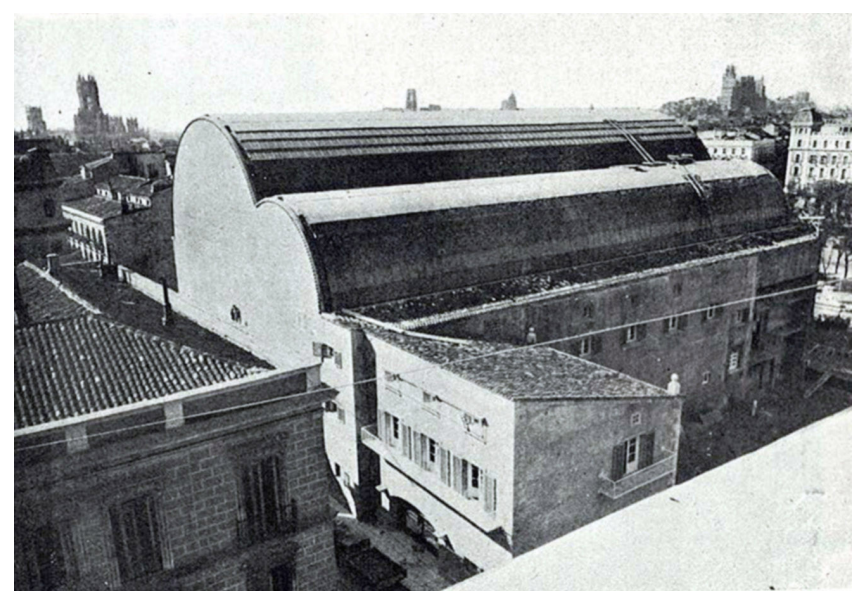

Figura 4. Inauguración el 29 de febrero de 1936. Obsérvese que falta por construir el volumen destinado a restaurante.

(Huarte y Cía, S. L. Construcciones. Frontón Recoletos. Volumen 5. Madrid: Tipografía Artística).

\section{EL CICLO VITAL: USOS Y ACTIVIDADES DEL FRONTÓN RECOLETOS}

Los usos que Zuazo y Torroja proponen en el proyecto del Frontón trascienden el ámbito del deporte para incorporar manifestaciones de carácter social y lúdico. 
La instalación deportiva desarrolla en el volumen principal un programa complejo. Se dispone una cancha con la correspondiente contracancha para el juego de la pelota vasca, junto con los espacios para los deportistas y otros auxiliares, de servicio, así como una zona de público con un aforo de 2.250 localidades. «Sus 2.250 asientos, se distribuían entre la planta de cancha, de palcos y de galería, y se complementaban con las llamadas localidades de "paseo" hasta un total de 2.850" (29). Estos usos se complementan con una sala de quinielas apuestas- y un bar, ubicados en la planta sótano y de palcos, respectivamente.

En el volumen, finalmente ejecutado después de la contienda civil española, situado en la esquina con la calle particular se disponen, en una secuencia sucesiva desde la planta inferior hasta la más alta, la cocina y sus servicios, el restaurante, la sala de té, y finalmente la vivienda del conserje. El otro volumen, conformando la esquina de las calles Villanueva y del Cid, acoge los accesos principales a la cancha de juego en la planta baja, así como las dependencias administrativas de la empresa NEFSA en las plantas superiores.

Y aunque en diversos textos se recoge la existencia de unos baños turcos en la planta sótano, el estudio de la documentación gráfica no ha podido corroborar su presencia. Entre ambos volúmenes, bajo la terraza-restaurante de verano, en el proyecto definitivo se dispone una bodega en la planta semisótano con acceso desde la calle Villanueva (Figura 5).

El proyecto, proponiendo diversas mejoras funcionales en las condiciones de confort ambiental, avanza un paso más en la evolución y transformación de los espacios deportivos especializados.

La puesta en funcionamiento del edificio que se produce en marzo de 1936, y aun considerando su incompleta construc- ción, asegura una adecuada utilización al lograr la autonomía funcional entre las distintas actividades (30), mediante un considerable número de escaleras definiendo circulaciones específicas, recorridos en algunos casos duplicados, en respuesta a las demandas de la época.

Las actividades deportivas que se venían celebrando en el Frontón desde su inauguración: partidos de pelota vasca, combates de boxeo y lucha libre..., se interrumpen con el comienzo de la guerra civil en julio de 1936. Desde las semanas iniciales de la contienda se usa como Hospital de sangre por iniciativa del Ateneo Libertario de Delicias, controlado por la FAI -Federación Anarquista Ibérica-.

Durante la guerra, en su entorno se producen numerosas explosiones, impactando sobre el edificio algunos proyectiles de cañón. Dos obuses en la primavera de 1937 ocasionan importantes aberturas en el faldón más grande de la cubierta provocando desplazamientos permanentes. La situación social y la guerra impiden controlar las deformaciones, ya que en el verano de 1936 se habían retirado los instrumentos de medición que registraban los movimientos de la cubierta. A pesar de todo ello, no se desmoronará hasta finalizada la guerra civil.

Acabado el conflicto en abril de 1939, la sociedad propietaria del inmueble manifiesta su intención de recuperarlo. Para ello, le encarga a Torroja el estudio y reparación de los daños ocasionados en el mismo. Este realiza un anteproyecto en el que propone recuperar el edificio dañado reforzando las láminas de la cubierta mediante unos anillos transversales sobre los lóbulos de hormigón armado hasta alcanzar los lucernarios (31).

Para efectuar una valoración del estado real de la cubierta, se instala un andamio y se despoja la bóveda de sus recubri-

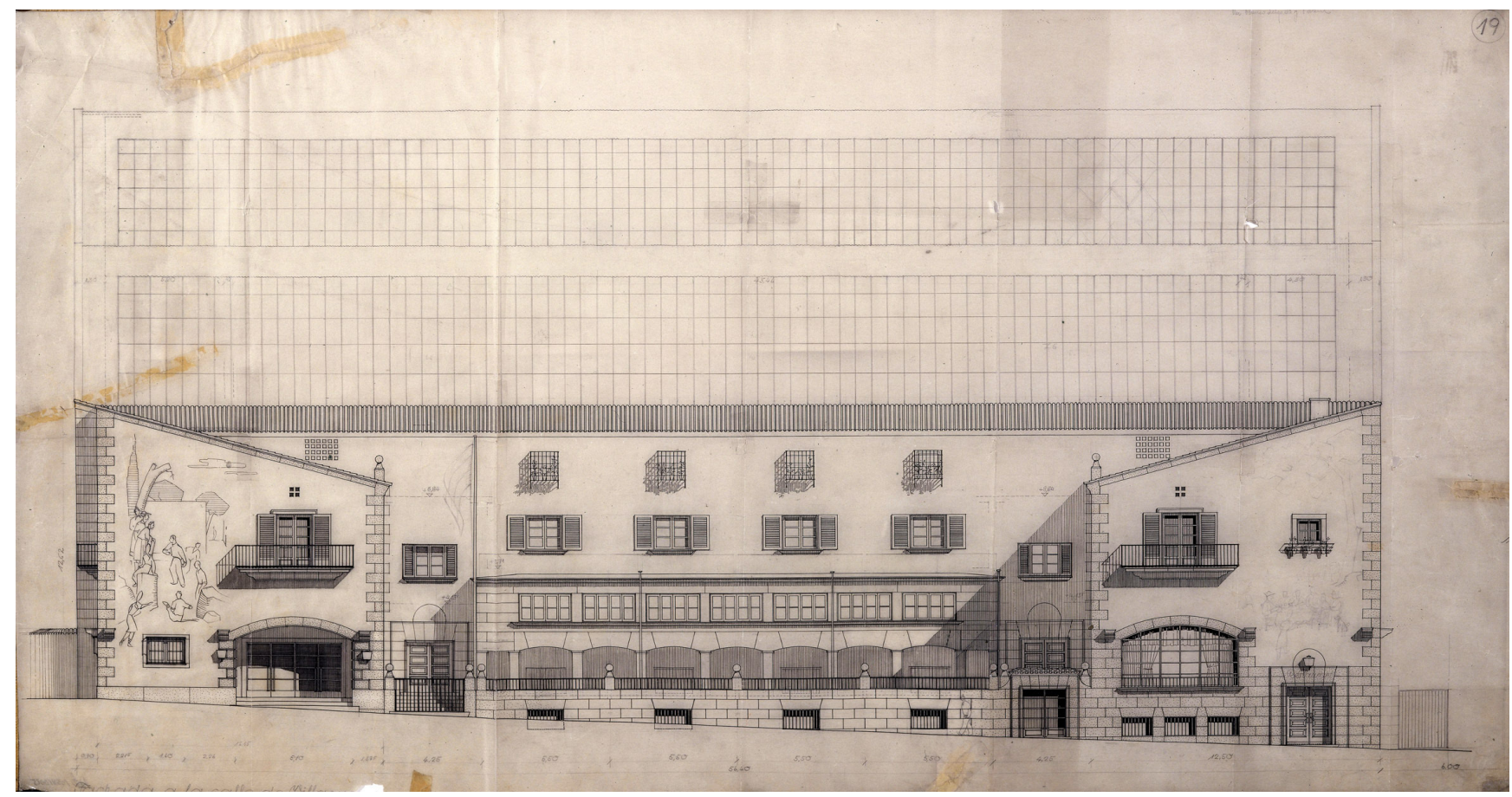

Figura 5. Proyecto de septiembre de 1935. Alzado a calle Villanueva.

(Fuente: Fondo Zuazo. Biblioteca Nacional de España). 
mientos. Encontrándose en este proceso, se produce el hundimiento de la cubierta el 15 de agosto de 1939, según refleja la Memoria del segundo ejercicio social (32), del año 1940, presentada por el Consejo de Administración de NEFSA ante la Junta General Ordinaria de accionistas.

El desplome, que ocasiona serios daños en el edificio, provoca que la empresa propietaria adopte con urgencia las medidas necesarias para restablecer el estado inicial de uso. Entre otras, estudia la posibilidad de reponer la cubierta laminar utilizando de nuevo la conocida cimbra de madera. Esta solución, con el país en un estado de reconstrucción general, se antoja en estos momentos antieconómica.

En esta coyuntura excepcional, NEFSA decide reconstruir la cubierta mediante un sistema de cerchas metálicas. Para ello, al encontrarse Zuazo expedientado y fuera del país ${ }^{1}$, le encarga el proyecto de reconstrucción del Frontón al arquitecto Felipe Heredero Igarza, quien lo elabora entre septiembre y octubre de 1939.

La reconstrucción no solamente alcanza la ejecución de una nueva cubierta metálica «sobre la base de conservar lo más aproximadamente la forma interior de la cubierta destruida (...) sin aumentar sensiblemente la altura total de la cubierta...» (33), sino que incluye las obras del pabellón del restaurante y sala de té conforme a los planos de Zuazo, ejecutándose el volumen que había quedado sin construir cuando se inauguró el edificio. Los cambios en la decoración y una inscripción con el nombre de F. Heredero como autor de este volumen encienden los ánimos de Pedro Zuazo, su hermano y voz en Madrid.

«Tengo que participarte para que me des instrucciones sobre la siguiente acción de tu sucesor y heredero de tus trabajos. Ha realizado la terminación de las obras proyectadas en el pabellón del restaurante de acuerdo con tus planos (...) y al arranque de la escalera, a la entrada en este pabellón por Villanueva, una alegoría con un pequeño recuadro al pie, en que perfectamente se lee aún desde la calle la inscripción RECOLETOS/ARQUITECTO: F. Heredero 1941. Tentado he estado de hacerme con galipot y dar unos brochazos pero...» (34).

La nueva cubierta se resuelve mediante una solución de doble cáscara; se independiza el espacio interior (Figura 6) del volumen exterior. Esta usual estructura y la forma rompen su vínculo más profundo. Pese a ello, la unidad espacial que se percibe en el interior del volumen continúa caracterizando la instalación deportiva.

Del mismo modo, la ubicación en el volumen menor de la calle Villanueva del restaurante y la sala de té confirma la intencionalidad de la visión sesgada del Paseo de Recoletos, alimentando el significado del Frontón como referencia urbana.

El Frontón Recoletos se reabre el 28 de marzo de 1940, «coincidiendo con la fecha del aniversario de la liberación de Madrid por el Glorioso Ejército Nacional» (32). La reconstrucción permite la reanudación de las actividades deportivas y sociales.

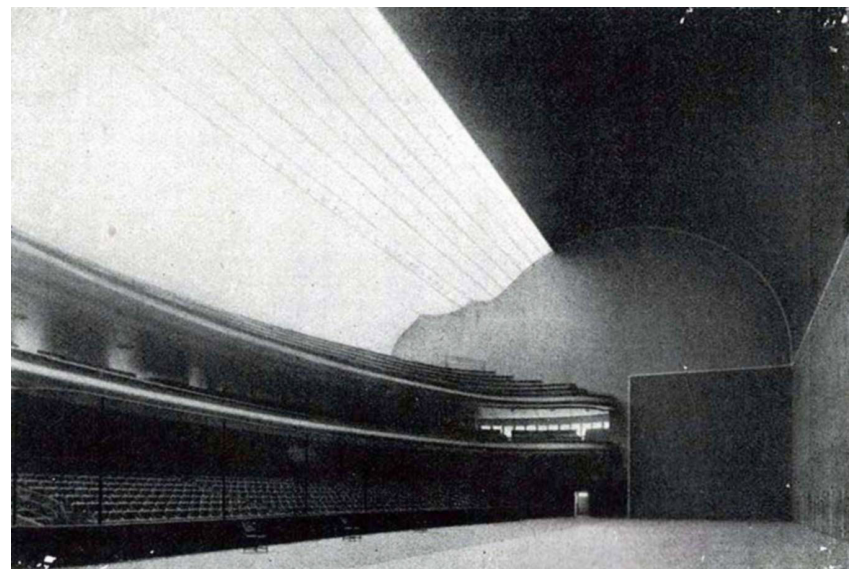

Figura 6. Reconstrucción (proyecto de F. Heredero Igarza). Interior, 1940 (Huarte y Cía, S. L. Construcciones. Frontón Recoletos. Volumen 5. Madrid: Tipografía Artística).

La práctica del deporte de la pelota vasca se contempla de manera específica, sin que ello impida la celebración de otras especialidades deportivas: boxeo, lucha libre, baloncesto.

Un plano de la planta del espacio útil deportivo, cancha y contracancha, con una distribución de un ring y de las correspondientes localidades a su alrededor, da cuenta de la utilización del Frontón para la actividad pugilística. Así mismo, un cartel anunciando la celebración de un encuentro amistoso de baloncesto y una vieja fotografía de este deporte revelan la utilización de una pista especializada por otra modalidad deportiva.

Entre los años 1940 y 1952 el Frontón es la primera pista cubierta, habilitándose como cancha adaptada, en la que celebra sus partidos de competición oficial la sección de baloncesto del Real Madrid, F.C.

La aparición del proyecto de un hotel en la superficie del solar no ocupada por el Frontón, fechado en marzo de 1952, evidencia el valor de centralidad urbana del lugar, aunque el proyecto no llega a ejecutarse.

El equipamiento deportivo continúa celebrando en la década de los años cincuenta eventos del mundo del deporte principalmente, sin renunciar a acomodar actos de índole social, tal y como venía haciendo.

«Se encomendó, sin embargo a nuestro Arquitecto un urgente estudio de la terraza y terminación consiguiente de las obras necesarias, en forma que permitiera la explotación de aquella; y el día 29 de junio (de 1940) fue abierta al público con el éxito extraordinario que es de todos conocido, hasta el extremo de haber llegado a ser durante el pasado verano el sitio de reunión en su género más selecto y concurrido de Madrid» (32).

Actividades dispares prosiguen en el Frontón hasta que fuertes intereses inmobiliarios provocan su completa demolición en 1973, para dar paso a un convencional edificio de viviendas. Con ello, el ciclo vital de cuatro décadas del Frontón Recoletos se extingue.

\footnotetext{
${ }^{1}$ Secundino Zuazo Ugalde abandona España en septiembre de 1936, estableciéndose en Francia durante la Guerra Civil. Al terminar esta, se le abre un expediente de Responsabilidades Políticas, y cuando retorna al país en julio de 1940, se le confina en las Islas Canarias hasta 1944.
} 


\section{CONCLUSIONES}

A pesar de su desaparición en 1973, y sin dejar en el olvido su rigor en el estricto orden económico ni su voluntad de responder servicialmente a la sociedad de su época, su significación como objeto arquitectónico de naturaleza esencial establece la íntima fusión de forma, función y estructura mediante un ejercicio gimnástico de equilibrio entre el alzado académico tradicional, la planta funcional y la sección tecnológica.

Sección tecnológica que determina, a través de un doble alarde estructural, el carácter representativo del edificio. Mientras que las láminas de hormigón armado son la manifestación evidente de uno de los principios invariantes de la arquitectura, salvar la mayor distancia posible con una luz estructural única mediante la menor cantidad de material posible, las vigas de gran canto con las ménsulas del graderío son el reflejo de la desmaterialización de los elementos físicos de soporte con una intencionada ocultación de la estructura intuida.
El diálogo entre las invariantes de la estructura formal profunda del Frontón Recoletos, independientemente de su apariencia expresiva, y el uso de un nuevo material, el hormigón armado, enfatiza la singularidad del modelo.

La ideación inicial del Frontón Recoletos como una sala deportiva especializada no impide que se celebren en ella actividades vinculadas a otros deportes, así como eventos de índole social. Así mismo, en determinados momentos de cambios sociales y políticos, se comporta como refugio de otros usos no previstos. Esta profusión usos le confiere un carácter multifuncional y polivalente.

Y aun cuando se gesta y se ejecuta en un breve período temporal, la presencia de la ingeniería en el paradigma arquitectónico desde el inicio de la ideación refleja la estrecha y fecunda colaboración del arquitecto Zuazo y el ingeniero Torroja. El manejo de los mecanismos de expresión, la contención de la forma y la volumétrica sobriedad estructural caracterizan una de las piezas arquitectónicas españolas más relevantes del siglo XX.

\section{REFERENCIAS}

(1) Diem, C. (1966). Historia de los deportes, vol. 1, p. 457 ( $1^{\mathrm{a}}$ ed.). Barcelona: Luis de Caralt.

(2) Diderot, D., d’Alambert, J. (1974). Encyclopédie de Diderot et d'Alambert. Dictionare Raisonné des Sciences, des Arts et des Metiers. París 1751-1772. En Recuil de planches sur les sciences, les arts liberaux et les arts mechániques, avec leur explication. Setieme livraison ou huitieme volume, 254 planches. Parma : Franco María Ricci. [A París, Chez Briasson, me Saint Jacques a la Science, 1771].

(3) Usón-García, R. (2004). La forma en la arquitectura deportiva. Visión retrospectiva y actual del problema estético. Ingeniería y Territorio, (66): 21.

(4) García-Gutiérrez Mosteiro, J. (2009). Frontones en Madrid (1891-1936). Singular tipo arquitectónico para la metrópoli. Ilustración de Madrid, (12):40.

(5) Machimbarrena-Gogorza, V. (1924). Arquitectura e Ingeniería. Revista de Obras Públicas, 72, tomo I (2397): 17-21

(6) Tarragó, S. (1979). La modernidad en la obra de Eduardo Torroja. Madrid: Colegio de Ingenieros de Caminos, Canales y Puertos/ Turner.

(7) Sambricio, C. (2008). La ingeniería en las revistas españolas de arquitectura: 1920-1936. Informes de la Construcción, 6o(510): 35-44, doi: http://dx.doi.org/10.3989/ic.2008.v6o.i510.727.

(8) Rodríguez-García, A., Hernando de la Cuerda, R. (2009). Timbrel Construction and Reinforced Concrete in Madrid Rationalism. En Proceedings of the Third International Congress on Construction History.

(9) Torroja, E. (1937-1938). Le voile mince du "Frontón Recoletos" à Madrid. Mémoires de l’AIPC, 5: 343-361, doi: http:// dx.doi.org/10.5169/seals-6167.

(10) Fullaondo-Errazu, J. D. (1970). Luz y sombra en la obra de Secundino Zuazo Ugalde. Arquitectura, (141): 38.

(11) Maure-Rubio, L. P. (1985). Secundino Zuazo: la arquitectura y el urbanismo en España en el primer tercio del siglo XX. (Tesis doctoral no publicada), p. 462. Madrid: Universidad Politécnica de Madrid.

(12) (1968). Frontón Recoletos. Nueva Forma, 32.

(13) Zuazo, S. (2003). Madrid y sus anhelos urbanísticos. Memorias inéditas de Secundino Zuazo 1919-1940. Sambricio, C. (Ed.). p. 294. Madrid: Dirección General de Urbanismo y Planificación Regional de la Comunidad de Madrid y Nérea.

(14) González-Varas Ibáñez, I. (2010). Los palacios de la Castellana, pp. 150-151. Madrid: Turner.

(15) Torroja-Miret, E. (1942). Memorias de la Real Academia de Ciencias Exactas, Físicas y Naturales de Madrid. Serie de Ciencias Exactas. En Comprobación y comportamiento de una estructura laminar (Tomo III) (pp. 12-13). Madrid: Real Academia de Ciencias Exactas, Físicas y Naturales de Madrid.

(16) Lozano-Galant, J. A., Payá-Zaforteza, I. (2011). Structural analysis of Eduardo Torroja’s Frontón de Recoletos' roof. Engineering Structures, 33(3): 843-854, doi: http://dx.doi.org/10.1016/j.engstruct.2010.12.006.

(17) De la Encina, J. (1933). Prólogo. En Arquitectura contemporánea en España, Tomo I. El arquitecto Zuazo Ugalde, p. IX. Madrid: Ediciones de Arquitectura y Urbanismo Edarba.

(18) Angoso, Á. (1936). Frontón Recoletos à Madrid. La Construction Moderne, 27: 821-827.

(19) Fernández-Ordóñez, J. A., Navarro-Vera, J. R. (1999). Eduardo Torroja Miret Ingeniero, pp. 53-55 y pp. 130-145. Madrid: Pronaos.

(20) Fullaondo, J. D. (1970). Luz y sombra en la obra de Secundino Zuazo Ugalde. Arquitectura, (141): 45.

(21) Larripa-Artieta, V. (2013). Frontón Recoletos: la construcción de la metáfora. Proyecto, Progreso, Arquitectura (Forma y Construcción en Arquitectura), (8): 72-87.

(22) Torroja, E. (1962). Frontón Recoletos. Informes de la Construcción, 14(137): 153-4.

(23) (1968). Frontón Recoletos. Nueva Forma, 35: 95-99. 
(24) (1936). Las estructuras del nuevo Frontón Recoletos (Madrid). La Construcción Moderna, 7: 49-50.

(25) Antuña-Bernardo, J. (2002). Las estructuras de edificación de Eduardo Torroja Miret, pp. 136-139. Madrid: Universidad Politécnica de Madrid - Escuela Técnica Superior de Arquitectura.

(26) Zuazo, S. (2003). Madrid y sus anhelos urbanísticos. Memorias inéditas de Secundino Zuazo 1919-1940, p. 327. Madrid: Dirección General de Urbanismo y Planificación Regional de la Comunidad de Madrid y Nérea.

(27) (1936). Notas diversas [Visita de técnicos a las obras del Frontón Recoletos y tribunas del nuevo Hipódromo]. La Construcción Moderna, 4: 30.

(28) Torroja-Miret, E. (1936). Cubiertas laminares de hormigón armado. Hormigón y Acero, 25: 173-185 (continuación de Hormigón y Acero, 24: 140-155).

(29) Maure-Rubio, L. (2004). Secundino Zuazo, Eduardo Torroja. Frontón Recoletos, Madrid 1935. Cuadernos de investigación de la Exposición de las Arquitecturas Ausentes del siglo XX, (24): 27.

(30) Staal, A. (1985). Reisbericht van Arthur Staal (Grand Prix de Rome 1936). En De 8 en Opbouw 1932-1943, Periodical of New Architecture, Part III, Vols. 1936 y 1937, pp. 218-222. [Reedición de Staal, A. (1936). Reisbericht van Arthur Staal (Grand Prix de Rome 1936). De 8 en Opbouw, 18].

(31) Antuña, J. (2004). The Evolution of the Work of Eduardo Torroja: Shell Roofs withand without Reinforcement Rings. En Second International Congress on Construction History, (pp. 179-194). Queens' College-Cambridge University.

(32) Memoria del segundo ejercicio social, año 1940. Junta General Ordinaria de señores accionistas de NEFSA (Nueva Empresa de Frontones, S.A.), 29 de marzo de 1941. Biblioteca Nacional de España - Fondo del arquitecto Secundino Zuazo, depositado en la carpeta identificada con la referencia exterior NEFSA.

(33) Heredero, F. (1939, octubre). Memoria del Proyecto de Reconstrucción del Frontón Recoletos. Archivo de la Villa de Madrid, expediente $n^{0} 45-9-22$.

(34) Correspondencia privada de P. Zuazo con su hermano S. Zuazo. Carta fechada el 17 de abril de 1941 en Madrid. Biblioteca Nacional de España - Fondo del arquitecto Secundino Zuazo, depositado en la carpeta identificada con la referencia exterior NEFSA. 